
КАЛИНИНГРАДСКОЙ ОБЛАСТИ С ОСТРЫМ КОРОНАРНЫМ СИНДРОМОМ

() Р.С. Богачев, А.Ю. Козел, Л.С. Литвинова, Л.В. Михайлова* Е.С. Шитова, В.Б. Анкудович, В.В. Мордвинцев, У.А. Добрынина

ФГАОУ ВО «Балтийский федеральный университет им. Иммануила Канта», Калининград, Россия

Обоснование. Витамин D-связывающий белок является основным переносчиком витамина D в крови, а также оказывает влияние на макрофагальное звено иммунитета. Доказана роль витамина D и макрофагов в патогенезе атеросклероза, но данных по витамин D-связывающему белку в этом отношении недостаточно.

Цель. Исследовать полиморфизм витамин D-связывающего белка у пациентов с острым коронарным синдромом (OKC) без сахарного диабета и определить взаимосвязь между аллелем витамин D-связывающего белка и особенностями ОКС у данной группы больных.

Материалы и методы. Исследование является одномоментным обсервационным. Объект исследования - больные с ОКС. Критерии исключения - сахарный диабет, аутоиммунные заболевания, злокачественные новообразования. Исследование проводилось в течение 5 мес, с ноября 2017 г. по март 2018 г. В исследование включены 50 пациентов, поступивших экстренно в стационар с диагнозом ОКС, из них 36 мужчин, 14 женщин. Средний возраст пациентов на момент включения в исследование 60 (55;66) лет. Всем обследованным была проведена оценка факторов риска сердечно-сосудистых заболеваний, общий и биохимический анализ крови, тропонины, коронарография, эхокардиография. Оценка распространенности полиморфизмов витамина D у данной группы пациентов с ОКС проводилась с помощью секвенирования по Сенгеру гена витамин D-связывающего белка. Для всех пациентов проводилось генотипирование однонуклеотидных полиморфизмов VDBP SNPs p.T436K (rs4588) и p.D432E (rs7041).

Результаты. Всего полиморфизм генов был выявлен у 43 из 50 (86\%) пациентов, включенных в исследование. Вариант аллеля Gc1s/2 (rs7041G-rs4588A) был найден у 7 (14\%) пациентов, Gc2 (rs7041T-rs4588A) - 9 (18\%) пациентов, Gc1s (rs7041G-rs4588C) - 20 (40\%) пациентов, Gc1f (rs7041T-rs4588C) - соответственно у 14 (28\%). По данным коронарографии: поражение коронарной артерии со стенозом более 50\% просвета сосуда было выявлено у 16 пациентов; стеноз более $90 \%$ просвета коронарного сосуда диагностирован у 8 пациентов, тотальная окклюзия коронарного сосуда - у 4 пациентов.

Заключение. Среди группы пациентов с ОКС встречаемость полиморфизма генов rs4588, и rs7041 была высокой и составила в нашем исследовании до 86\%. При распределении пациентов по группам в соответствии с выявленным вариантом аллеля гена VDBP выявлено, что особенностью группы пациентов с вариантом аллеля VDBP Gc2 являлась относительно высокая частота повторных инфарктов миокарда и тотальной окклюзии коронарных артерий, а также тенденция к более низкому уровню витамина $\mathrm{D}_{3}(25(\mathrm{OH}) \mathrm{D})$ в сыворотке крови.

КЛЮЧЕВЫЕ СЛОВА: витамин D-связывающий белок; острый коронарный синдром; полиморфизм витамин D-связывающего белка; витамин D; атеросклероз; артериальная гипертензия.

\title{
VITAMIN D BINDING PROTEIN POLYMORPHYSM IN PATIENTS WITH ACUTE CORONARY SYNDROME IN KALININGRAD REGION
}

(c) Robert S. Bogachev, Anastasia Y. Kozel, Larisa S. Litvinova, Larisa V. Mikhailova*, Elena S. Shytova, Vitaly B. Ankudovich, Vladislav V. Mordvintsev, Ulyana A. Dobrynina

Immanuel Kant Baltic Federal University, Kaliningrad, Russia

BACKGROUND: Vitamin D binding protein is a main vitamin D carrier in serum. It also has an impact on macrophagial function. Role of vitamin $D$ and macrophages in the pathogenesis of atherosclerosis is scientifically proven but there is lack of data on vitamin $D$ binding protein in this regard.

AIMS: To evaluate the vitamin D binding protein polymorphism in patients with acute coronary syndrome without diabetes mellitus, autoimmune diseases and malignant tumors. Determine correlation, if there is, between vitamin $D$ binding protein allele and features of acute coronary syndrome among this patient group.

MATERIALS AND METHODS: It is a cross-sectional observational study. Study subjects are patients with acute coronary syndrome. Exclusion criteria are the presence of diabetes mellitus, autoimmune diseases and malignant tumors. In all participants were evaluated: predisposing factors for heart diseases, CBC, biochemical blood test, troponin, coronarography, echocardiography. The study lasted for 5 months from November 2017 until March 2018. Primary end point - assessment of vitamin D binding protein polymorphysm in this group of patients with acute coronary syndrome by means of vitamin $\mathrm{D}$ binding protein gene sequencing. 50 patients were enrolled into this study who were urgently admitted to hospital 
and diagnosed with acute coronary syndrome. Among them - 36 males and 14 females. Mean age was 60 (55;66) years. All participants were sequenced for single nucleotide polymorphysm in VDBP p.T436K ( $r$ 4588) and P.432E (rs7041).

RESULTS: Gene polymorphysms of interest were found in 43 patients among 50 enrolled. Haplotype Gc1s/2 (rs7041G-rs4588A) was found in 7 (14\%) patients, Gc2 (rs7041T-rs4588A) — in 9 (18\%) patients, Gc1s (rs7041G-rs4588C) in $20(40 \%)$ patients, Gc1f (rs7041T-rs4588C) in 14 (28\%). Coronarography showed that coronary artery occlusions obstructing more than $50 \%$ of vessel lumen was found in 16 patients; obstruction greater than $90 \%$ was seen in 8 patients; total occlusion - in 4 patients.

CONCLUSIONS: In patient group with acute coronary syndrome prevalence of vitamin D binding protein gene polymorphysm was high - in $86 \%$ of participants. The features of Gc2 haplotype were higher frequency of recurrent myocardial infarction and total coronary artery occlusion, as well as tendency to decreased serum vitamin D3 (25(OH)D) levels.

KEYWORDS: vitamin D binding protein; acute coronary syndrome; vitamin D binding protein polymorphysm; vitamin D; atherosclerosis.

\section{ОБОСНОВАНИЕ}

Низкий уровень метаболитов витамина D $(25(\mathrm{OH}) \mathrm{D}$ и 1,25(OH) 2 D) ассоциирован с повышением риска сердечно-сосудистых событий [1-7]. Морфологическим субстратом для данных событий является атерома интимы сосудов. На данный момент ведущая роль в атерогенезе отводится воспалению. Мигрировавшие в интиму сосудов моноциты и другие лейкоциты синтезируют широкий спектр провоспалительных цитокинов, выполняя ключевую роль в прогрессии атеросклероза [8]. Антиатерогенные эффекты витамина D достигаются за счет регуляции иммунологических/воспалительных реакций [9]. Витамин D ингибирует субъединицу р22 НАДФН-оксидазы, улучшает димеризацию эндотелиальной NO-синтазы (eNOS), что способствует синтезу NO и снижению образования активных форм кислорода. 1,25(OH), D блокирует сигнальный путь NF-kB, в результате чего снижается экспрессия провоспалительных цитокинов ИЛ-6,-8, RANTES, молекул клеточной адгезии ICAM-1, VCAM-1, PECAM-1 и Е-селектина. За счет снижения экспрессии циклооксигеназы-2 и повышения экспрессии 15-гидроксипростагландин-дегидрогеназы, снижается уровень простагландинов и части других провоспалительных цитокинов [10]. Также витамин D оказывает влияние на ренин-ангиотензин-альдостероновую систему, снижая артериальное давление [11].

Большая часть (85-90\%) 25(OH)D и 1,25(OH), D связана с витамин D-связывающим белком (VDBP), меньшая часть (10-15\%) - с альбумином, и около $1 \%$ находится в несвязанном виде $[12,13]$. Считается, что связанные фракции недоступны для клеток $[14,15]$. VDBP играет важную роль не только в непосредственной транспортировке витамина $D$, но также имеет большое количество иных функций. VDBP связывает свободный актин, транспортирует жирные кислоты $[15,16]$. Доказана роль участия VDBP в иммунных реакциях в качестве активатора макрофагального звена иммунитета [17]. Было предположено, что различные полиморфизмы оказывают разное влияние на активацию макрофагов [18]. Известно, что VDBP становится макрофагальным активирующим фактором лишь после дегликозилирования с помощью галактозидаз и сиалидаз Т- и В-лимфоцитов. С этой позиции особенно выделяется Gc2 форма VDBP, которая может быть гликозилирована только в одной 418 позиции в отличие от Gc1, которая может быть гликозилирована по двум позициям - 418 и 420. Соответственно, после частичного дегликозилирования продукт Gc2 варианта VDBP имеет сниженную активность в отношении активации макрофагов [19].

VDBP имеет около 124 вариантов аллелей, при этом самыми распространенными являются полиморфизмы Gc1*1F, Gc*1S, Gc*2 [20]. Различные варианты данного белка имеют разную активность в отношении выполняемых им функций [21].

\section{ЦЕЛЬ}

Исследовать полиморфизм витамин D-связывающего белка у пациентов с ОКС без сахарного диабета и определить взаимосвязь между аллелем витамин D-связывающего белка и особенностями ОКС у данной группы больных.

\section{МЕТОДЫ}

\section{Дизайн исследования}

Одномоментное обсервационное одноцентровое неконтролируемое исследование.

\section{Критерии соответствия}

Критерием включения явилось наличие диагноза «острый коронарный синдром» при поступлении. Критериями исключения в исследование явилось наличие сахарного диабета, аутоиммунных заболеваний и злокачественных новообразований, подтвержденных клинически.

\section{Условия проведения}

Исследование проведено на базе ГБУЗ «ОКБ Калининградской области». Все пациенты являлись жителями Калининградской области - региона с низкой инсоляцией.

Продолжительность исследования

Исследование проведено в период с ноября 2017 г. по март 2018 г.

Объекты (участники) исследования

В исследование включено 50 пациентов - жителей Калининградской области, с диагнозом ОКС, из них 36 (72\%) мужчин и 14 (28\%) женщин. Средний возраст пациентов составил $60(55 ; 66)$ лет. Самому молодому пациенту на момент включения в исследование было 42 года, самому пожилому - 83 года. Доля пациентов среднего 
возраста (от 45 до 60 лет) составила 50\% (25 пациентов), пожилого возраста (старше 60 лет) - 44\% (22 пациента), молодого возраста (до 45 лет) - 6\% (3 пациента).

При расспросе впервые возникшие боли за грудиной отметили 32 (64\%) пациента. Диагноз ишемической болезни сердца (ИБС) до момента включения в исследование был выставлен 18 (36\%) пациентам, из них 12 (24\%) ранее перенесли инфаркт миокарда. Длительность заболевания ИБС в среднем составила 14 мес. ОКС с подъемом сегмента ST был диагностирован у 13 (26\%) пациентов.

Артериальная гипертензия наблюдалась у 44 (88\%) пациентов, из них артериальная гипертензия 3 степени была диагностирована у 35 (70\%) пациентов, 2 степени у $6(12 \%)$ пациентов.

Избыточная масса тела была выявлена у 20 (40\%) пациентов, ожирение I степени - у 8 (16\%) пациентов, ожирение II степени - у 2 (4\%) пациентов.

\section{Описание медицинского вмешательства}

Эхокардиографическое исследование проводилось на ультразвуковом аппарате Samsung Medison Accuvix XG. Протокол эхокардиографического исследования включал расчет индекса массы миокарда левого желудочка (ИММЛЖ), фракция выброса (ФВ) левого желудочка рассчитывалась по формуле Teichholz. Селективная коронароангиография выполнялась на ангиографе General Electric Innova 3100 IQ с использованием контрастного вещества Омнипак.

Исследование уровня витамина $\mathrm{D}_{3}(25(\mathrm{OH}) \mathrm{D})$ в сыворотке крови проводилось методом иммуноферментного анализа с использованием фотометра для микропланшетов Model 680 Microplate Reader (Bio-Rad, Hercules, CA, USA) и автоматизированной станции для мойки микропланшетов Bio-Plex Pro II Microplate Wash Station (Bio-Rad, Hercules, CA, USA).

Генотипирование производилось по следующей методике: геномная ДНК была выделена из замороженной цельной крови (хранившейся при $-20^{\circ} \mathrm{C}$ в вакутейнерах с К3 ЭДТА) с использованием набора реагентов GeneJet для выделения геномной ДНК из цельной крови (Thermo Fischer, Waltham, МА, США) в соответствии с протоколом производителя. Генотипирование двух однонуклеотидных полиморфизмов VDBP SNPs p.T436K (rs4588) и p.D432E (rs7041) производилось с помощью секвенирования. Амплификацию фрагментов размером 350-bp проводили, используя следующие праймеры: VDBP-F: 5'-GGCA TGTTTCACTTTCTGATCTC-3' и VDBP-R: 5'-TGCCATGTTAAGTGGAGGGT-3'. ПЦР проводили в амплификаторе T100 Thermal Cycler (Bio-Rad, Hercules, CA, USA). Амплификацию ДНК проводили в реакционной смеси, используя смесь для ПЦР Master Mix с Таq ДНК-полимеразой (2,5 е.а./мкл) (Thermo Fischer, Waltham, MA, USA) в следующих условиях: начальная денатурация 1 мин $95^{\circ} \mathrm{C}$, далее 30 циклов - 30 с при $95^{\circ} \mathrm{C}$, отжиг праймеров $58^{\circ} \mathrm{C} 30$ с и 30 с элонгация при $72^{\circ} \mathrm{C}$; далее последний цикл синтеза 5 мин $72^{\circ} \mathrm{C}$. После очищения ДНК путем реакции с ExoSap, циклический сиквенс производился с использованием набора BigDye ${ }^{\circledR}$ Terminator v3.1 Cycle Sequencing Kit. Далее нуклеотидная последовательность определялась на секвенаторе Hitachi 3730 Genetic Analyzer и была проанализирована с помощью программного обеспечения SnapGene, version 4.1 (GSL Biotech LLC).

\section{Основной исход исследования}

Выявление полиморфизма гена витамин D-связывающего белка и оценка влияния его на клиническое течение ОКС.

\section{Этическая экспертиза}

Все пациенты подписали информированное согласие.

Решение комиссии по этике Балтийского федерального университета им. И. Канта: утвердить положительное заключение о возможности проведения исследования «Оценка полиморфизма гена витамин Д-связывающего белка, дефицита витамина D и ионных нарушений у лиц старше 50 лет с выраженным атеросклерозом и их связь с возникновением сердечно-сосудистых событий у данной категории пациентов - жителей Калининградской области».

Протокол заседания комиссии по этике БФУ им. И. Канта № 5 от 29.06.2018.

\section{Статистический анализ}

Принципы расчета размера выборки: размер выборки предварительно не рассчитывался.

Методы статистического анализа данных: статистический анализ полученных данных проводился с использованием пакета прикладных статистических программ StatSoft STATISTICA 7,0. Непрерывные переменные представлены в виде медианы и межквартильных интервалов (Ме (25\%;75\%). Номинальные данные представлены в виде относительных частот объектов исследования (n, \%).

\section{РЕЗУЛЬТАТЬ}

Основные результаты исследования

В качестве критериев дефицита витамина D использованы критерии Российской ассоциации эндокринологов, 2015 [22]. Дефицит витамина D (уровень ниже 20 нг/мл) выявлен у 41 (82\%), из них критически низкий уровень (менее 10 нг/мл) - у 3 (7\%) пациентов, недостаточность витамина D (уровень витамина 21-29 нг/мл) - у 8 (16\%), достаточное содержание витамина D (более >30 нг/мл) у $1(2 \%)$ пациента.

При генотипировании однонуклеотидных полиморфизмов VDBP SNPs p.T436K (rs4588) и p.D432E (rs7041) методом секвенирования по Сенгеру полиморфизм генов был выявлен у 43 (86\%) из 50 пациентов, включенных в исследование. Полиморфизм rs4588 (C>A) выявлен у 16 (32\%) из 50 пациентов с ОКС. Полиморфизм rs7041(T>G) был выделен у 27 (54\%) из 50 пациентов с ОКС. Полиморфизм rs4588 и rs7041 - у 7 (14\%) пациентов, включенных в исследование.

Пациенты были распределены по группам в соответствии с выявленным вариантом аллеля гена VDBP. Вариант аллеля Gc1s/2 (rs7041G-rs4588A) был найден у 7 (14\%) пациентов, Gc2 (rs7041T-rs4588A) - 9 (18\%) пациентов, Gc1s (rs7041G-rs4588C) - 20 (40\%) пациентов, Gc1f (rs7041T-rs4588C) - соответственно у 14 (28\%). Основные характеристики групп представлены в таблице 1. 
Таблица 1. Основные характеристики групп

\begin{tabular}{|c|c|c|c|c|}
\hline Показатели & Gc1s/2, n=7 & Gc2, n=9 & Gc1s, $n=20$ & Gc1f, $n=14$ \\
\hline Возраст, Me (25\%;75\%) & $58,9(55,5 ; 66)$ & $62(57 ; 64)$ & $58(53 ; 62)$ & $62,4(54 ; 69)$ \\
\hline Мужчины, n (\%) & $5(71,5)$ & $5(56)$ & $12(60)$ & $14(100)$ \\
\hline Женщины, n (\%) & $2(28,5)$ & $4(44)$ & $8(40)$ & $0(0)$ \\
\hline $\mathrm{A} \Gamma, \mathrm{n}(\%)$ & $5(71)$ & $9(100)$ & $17(85)$ & $13(93)$ \\
\hline 1 степень & - & - & - & $2(15)$ \\
\hline 2 степень & - & $3(33)$ & $1(6)$ & $2(15)$ \\
\hline 3 степень & $4(80)$ & $6(67)$ & $16(94)$ & $9(70)$ \\
\hline ИМ в анамнезе, n (\%) & $2(29)$ & $3(33)$ & $4(25)$ & $2(15)$ \\
\hline Ожирение, n (\%) & - & $3(33)$ & $3(15)$ & $4(29)$ \\
\hline Избыточный вес, n (\%) & $3(43)$ & $3(33)$ & $9(45)$ & $5(36)$ \\
\hline Общий холестерин, ммоль/л & $6,1(5,1 ; 6,6)$ & $4,75(4,0 ; 5,5)$ & $6,0(4,8 ; 6,85)$ & $6,0(4,9 ; 6,3)$ \\
\hline Уровень витамина D, нг/мл & $17,2(12,3 ; 21,5)$ & $13,3(13,0 ; 16,8)$ & $15,7(12,9 ; 17,9)$ & $16,7(12,85 ; 20,73)$ \\
\hline Подъем сегмента ST, n (\%) & $1(14)$ & $2(22)$ & $8(40)$ & $2(14)$ \\
\hline Тропонин+, n (\%) & $3(43)$ & $3(33)$ & $13(65)$ & $10(72)$ \\
\hline Гипертрофия левого желудочка, n (\%) & $2(29)$ & $3(38)$ & $14(70)$ & $9(69)$ \\
\hline ИММЛЖ, г/м² & $112(96 ; 114)$ & $106(88 ; 125)$ & $118(108 ; 132)$ & $118(102 ; 136)$ \\
\hline Снижение фракции выброса <45\%, n (\%) & $2(29)$ & $2(22)$ & $4(25)$ & - \\
\hline Окклюзия коронарных артерий >50\%, n (\%) & $3(43)$ & $2(22)$ & $6(30)$ & $5(36)$ \\
\hline Окклюзия коронарных артерий >90\%, n (\%) & $2(29)$ & $2(22)$ & $3(15)$ & $2(14)$ \\
\hline Тотальная окклюзия коронарных артерий, n (\%) & $1(14)$ & $2(22)$ & $1(5)$ & - \\
\hline
\end{tabular}

Группы больных не различались по возрасту. В группе с гаплотипом Gc1f, который считается самым благоприятным, относительно чаще встречались мужчины. Статистически значимых различий по степени АГ между группами не выявлено, однако у всех пациентов из группы с вариантом аллеля Gc2 была выявлена АГ. Повторный инфаркт миокарда чаще встречался у пациентов из групп с выявленным интересующим полиморфизмом, т. е. Gc1s/2, Gc2, Gc1s - 29\%, 33\% и 25\% соответственно. Ожирение чаще встречалось у пациентов с гаплотипами Gc2 (33\%), Gc1f (29\%), однако избыточный вес был выявлен у пациентов из групп Gc1s/2 (43\%) и Gc1s (45\%). Наименьший уровень холестерина выявлен у группы Gc2 - 4,75(4,0;5,5) ммоль/л, что можно объяснить веро- ятным приемом статинов и большей комплаентностью к лечению данной группы пациентов с АГ на фоне перенесенного ИМ, хотя оценка лечения не входила в задачи нашего исследования.

Гипертрофия левого желудочка чаще выявлялась у больных из групп Gc1s и Gc1f - 70\% и 69\% соответственно. Наиболее выраженное поражение коронарных артерий было выявлено у больных с гаплотипом Gc2, включая тотальную окклюзию коронарных артерий.

В группах пациентов с гаплотипами Gc2 и Gc1s у большинства был выявлен дефицит витамина D - 89\% и 90\% соответственно (табл. 2), однако наименьший средний уровень витамина D выявлен у лиц с вариантом аллеля Gc2 13,3 (13,0;16,8) нг/мл.

Таблица 2. Уровень витамина D в группах, n (\%)

\begin{tabular}{lccc}
\hline Вариант аллеля & $\begin{array}{c}\text { Дефицит витамина D } \\
\mathbf{n = 4 1}\end{array}$ & $\begin{array}{c}\text { Недостаточность витамина D } \\
\mathbf{n = 8}\end{array}$ & $\begin{array}{c}\text { Достаточное содержание } \\
\text { витамина D, } \mathbf{n = 1}\end{array}$ \\
\hline $\mathrm{Gc1s} / 2, \mathrm{n}=7$ & $5(71)$ & $2(29)$ & - \\
$\mathrm{Gc} 2, \mathrm{n}=9$ & $8(89)$ & $1(11)$ & - \\
$\mathrm{Gc1s}, \mathrm{n}=20$ & $18(90)$ & $1(5)$ & $1(5)$ \\
$\mathrm{Gc1f}, \mathrm{n}=14$ & $10(71)$ & $4(29)$ & - \\
\hline
\end{tabular}




\section{ОБСУЖДЕНИЕ}

На данный момент имеется ограниченное количество работ, связывающих витамин D связывающий белок VDBP с предрасположенностью, течением и особенностями различных заболеваний. В одной работе показано снижение частоты развития бронхолегочной дисплазии у пациентов с Gc2 вариантом VDBP [23]. В другом исследовании предположено, что Gc*1F вариант может быть фактором риска развития хронической обструктивной болезни легких (ХОБЛ) [24]. Есть данные о том, что данный белок является фактором риска развития метаболического синдрома, который, как известно, осложняется ранними сердечно-сосудистыми заболеваниями [25]. На сегодняшний день отсутствуют какие-либо данные, позволяющие связать полиморфизмы VDBP с особенностями течения острого коронарного синдрома. В нескольких исследованиях показано, что полиморфизмы rs7041 и rs4588 не связаны ни с уровнем витамина D в крови, ни с объемом поражения коронарных артерий атеросклерозом. В данных исследованиях эта связь была доказана лишь для самого витамина D [26-29]. Однако результаты относительно недавно проведенного в Китае популяционного исследования указывают на то, что дефицит витамина D чаще выявлялся у лиц с Gc2 вариантом гена VDBP [30]. Учитывая небольшой размер выборки пациентов, статистически значимых различий между группами получено не было. Однако нами было показано, что у лиц с ОКС чаще встречается дефицит витамина $D$, а также тенденция к более низкому его уровню у пациентов с вариантом аллеля Gc2. K тому же среди пациентов данной группы относительно чаще встречалось ожирение, тотальная окклюзия коронарных артерий и предшествующие инфаркты миокарда в анамнезе. В связи с этим можно предположить, что Gc2 вариант является прогностически более неблагоприятным для течения ИБС. Для подтверждения этого предположения необходимы дальнейшие исследования.

\section{ЗАКЛЮЧЕНИЕ}

Таким образом, среди группы пациентов с ОКС частота полиморфизма гена Gc rs4588 и rs7041 была высокой и составила в нашем исследовании 86\%. У 82\% пациентов среднего и пожилого возраста, поступивших в стационар с ОКС, был диагностирован дефицит витамина D. Особенностью группы пациентов с вариантом аллеля VDBP Gc2 являлась относительно высокая частота повторных инфарктов миокарда и тотальной окклюзии коронарных артерий, а также тенденция к более низкому уровню витамина $\mathrm{D}_{3}(25(\mathrm{OH}) \mathrm{D})$ в сыворотке крови.

\section{ДОПОЛНИТЕЛЬНАЯ ИНФОРМАЦИЯ}

Источник финансирования. Исследование выполнено при поддержке программы 5-100 «Проект повышения конкурентоспособности ведущих российских университетов среди ведущих мировых научно-образовательных центров» на базе ФГАОУ «БФУ им. И Канта».

Конфликт интересов. Авторы декларируют отсутствие явных и потенциальных конфликтов интересов, связанных с публикацией настоящей статьи.

Участие авторов. Богачев Роберт Стефанович - формирование плана и структуры исследования, проведение анализа полученных клинических и параклинических данных; Литвинова Лариса Сергеевна - исследование уровня 25-ОН витамина D методом иммуноферментного анализа; Козел Анастасия Юрьевна - генотипирование однонуклеотидных полиморфизмов VDBP SNPs p.T436K (rs4588) и p.D432E (rs7041) методом секвенирования, анализ полученных данных; Шитова Елена Сергеевна проведение статистического анализа; Михайлова Лариса Викторовна проведение статистического анализа; Анкудович Виталий - набор пациентов, анкетирование, проведение физикального обследования, обзор литературных данных; Мордвинцев Владислав Валерьевич - набор пациентов, анкетирование, проведение физикального обследования, обзор литературных данных; Добрынина Ульяна Андреевна - набор пациентов, забор биологического материала для исследования, статистическая обработка данных. Все авторы внесли значимый вклад в исследование и подготовку статьи. Авторы прочли и одобрили финальную версию статьи перед публикацией.

\section{СПИСОК ЛИТЕРАТУРЫ | REFERENCES}

1. Hutchinson MS, Grimnes G, Joakimsen RM, et al. Low serum 25-hydroxyvitamin D levels are associated with increased all-cause mortality risk in a general population: the Tromsø study. Eur J Endocrinol. 2010;162(5):935-942. doi: https://doi.org/10.1530/EJE-09-1041

2. Liu L, Chen M, Hankins SR, et al. Serum 25-Hydroxyvitamin D Concentration and Mortality from Heart Failure and Cardiovascular Disease, and Premature Mortality from All-Cause in United States Adults. Am J Cardiol. 2012;110(6):834-839. doi: https://doi.org/10.1016/j.amjcard.2012.05.013

3. de Boer $\mathbb{H}$. Serum 25-Hydroxyvitamin D Concentration and Risk for Major Clinical Disease Events in a Community-Based Population of Older Adults. Ann Intern Med. 2012;156(9):627. doi: https://doi.org/10.7326/0003-4819-156-9-201205010-00004

4. Kestenbaum B, Katz R, de Boer I, et al. Vitamin D, Parathyroid Hormone, and Cardiovascular Events Among Older Adults. J Am Coll Cardiol. 2011;58(14):1433-1441. doi: https://doi.org/10.1016/j.jacc.2011.03.069

5. Melamed ML, Michos ED, Post W, Astor B. 25-Hydroxyvitamin D Levels and the Risk of Mortality in the General Population. Arch Intern Med. 2008;168(15):1629-1637. doi: https://doi.org/10.1001/archinte.168.15.1629
6. Dobnig H. Independent Association of Low Serum 25-Hydroxyvitamin D and 1,25-Dihydroxyvitamin D Levels With All-Cause and Cardiovascular Mortality. Arch Intern Med. 2008;168(12):1340-1349. doi: https://doi.org/10.1001/archinte.168.12.1340

7. Robinson-Cohen C, Hoofnagle AN, Ix JH, et al. Racial Differences in the Association of Serum 25-Hydroxyvitamin D Concentration With Coronary Heart Disease Events. JAMA. 2013;310(2):179. doi: https://doi.org/10.1001/jama.2013.7228

8. Geovanini GR, Libby P. Atherosclerosis and inflammation: overview and updates. Clin Sci. 2018;132(12):1243-1252. doi: https://doi.org/10.1042/CS20180306

9. Agrawal D, Yin K. Vitamin D and inflammatory diseases. J Inflamm Res. May 2014:69. doi: https://doi.org/10.2147/JIR.S63898

10. Kassi E, Adamopoulos C, Basdra EK, Papavassiliou AG. Role of Vitamin D in Atherosclerosis. Circulation. 2013;128(23):2517-2531. doi: https://doi.org/10.1161/CIRCULATIONAHA.113.002654

11. LiYC. Vitamin D regulation of the renin-angiotensin system. J Cell Biochem. 2003;88(2):327-331. doi: https://doi.org/10.1002/jcb.10343

12. Bikle DD, Siiteri PK, Ryzen E, et al. Serum Protein Binding of 1,25-Dihydroxyvitamin D: A Reevaluation by Direct Measurement of Free Metabolite Levels*. J Clin Endocrinol Metab. 1985;61 (5):969-975. doi: https://doi.org/10.1210/jcem-61-5-969 
13. Safadi FF, Thornton P, Magiera $\mathrm{H}$, et al. Osteopathy and resistance to vitamin $\mathrm{D}$ toxicity in mice null for vitamin $\mathrm{D}$ binding protein. J Clin Invest. 1999;103(2):239-251. doi: https://doi.org/10.1172/JCI5244

14. Bikle DD, Gee E. Free, and Not Total, 1,25-Dihydroxyvitamin D Regulates 25-Hydroxyvitamin D Metabolism by Keratinocytes. Endocrinology. 1989;124(2):649-654. doi: https://doi.org/10.1210/endo-124-2-649

15. Mc Leod JF, Kowalski MA, Haddad JG. Interactions among serum vitamin D binding protein, monomeric actin, profilin, and profilactin. J Biol Chem. 1989;264(2):1260-1267. PMID: 2910852

16. Williams MH, Van Alstyne EL, Galbraith RM. Evidence of a novel association of unsaturated fatty acids with Gc (Vitamin D-binding protein). Biochem Biophys Res Commun. 1988;153(3):1019-1024 doi: https://doi.org/10.1016/S0006-291X(88)81330-5

17. Nagasawa H, Uto Y, Sasaki H, et al. Gc protein (vitamin D-binding protein): Gc genotyping and GcMAF precursor activity. Anticancer Res. 2005;25(6A):3689-3695. PMID: 16302727

18. Wood AM, Bassford C, Webster D, et al. Vitamin D-binding protein contributes to COPD by activation of alveolar macrophages. Thorax. 2011;66(3):205-210. doi: https://doi.org/10.1136/thx.2010.140921

19. Martineau AR, Leandro ACCS, Anderson ST, et al. Association between Gc genotype and susceptibility to TB is dependent on vitamin D status. Eur Respir J. 2010;35(5):1106-1112. doi: https://doi.org/10.1183/09031936.00087009

20. Cleve H, Constans J. The Mutants of the Vitamin-D-Binding Protein: More than 120 Variants of the GC/DBP System. Vox Sang. 1988;54(4):215-225. doi: https://doi.org/10.1111/j.1423-0410.1988.tb03908.x

21. Arnaud J, Constans J. Affinity differences for vitamin D metabolites associated with the genetic isoforms of the human serum carrier protein (DBP). Hum Genet. 1993;92(2). doi: https://doi.org/10.1007/BF00219689

22. Пигарова Е.А., Рожинская Л.Я., Белая Ж.Е., и др. Клинические рекомендации Российской ассоциации эндокринологов по диагностике, лечению и профилактике дефицита витамина D у взрослых // Проблемы Эндокринологии. - 2016. - Т. 62. - №4. - C. 60-84. [Pigarova EA, Rozhinskaya LY, Belaya JE, et al. Russian Association of Endocrinologists recommendations for diagnosis, treatment and prevention of vitamin D deficiency in adults. Problems of Endocrinology. 2016.62(4):60-84. doi: https://doi.org/10.14341/probl201662460-84

23. Serce Pehlevan O, Karatekin G, Koksal V, et al. Association of vitamin $\mathrm{D}$ binding protein polymorphisms with bronchopulmonary dysplasia: a case-control study of ge globulin and bronchopulmonary dysplasia. J Perinatol. 2015;35(9):763-767. doi: https://doi.org/10.1038/jp.2015.58

24. Ishii T, Keicho N, Teramoto S, et al. Association of Gc-globulin variation with susceptibility to COPD and diffuse panbronchiolitis. Eur Respir J. 2001;18(5):753-757. doi: https://doi.org/10.1183/09031936.01.00094401

25. Santos BR, Lecke SB, Spritzer PM. Genetic variant in vitamin D-binding protein is associated with metabolic syndrome and lower 25-hydroxyvitamin D levels in polycystic ovary syndrome: A cross-sectional study. PLoS One. 2017;12(3):e0173695. doi: https://doi.org/10.1371/journal.pone.0173695

26. Daffara $V$, Verdoia $M$, Rolla R, et al. Impact of polymorphism rs7041 and rs4588 of Vitamin D Binding Protein on the extent of coronary artery disease. Nutr Metab Cardiovasc Dis. 2017;27(9):775-783. doi: https://doi.org/10.1016/j.numecd.2017.06.002

27. Skaaby T, Thuesen BH, Linneberg A. Vitamin D, Cardiovascular Disease and Risk Factors. In: Pharmacopsychiatry. Vol 52. Drug Discovery. Cambridge: Royal Society of Chemistry; 2017:221-230. doi: https://doi.org/10.1007/978-3-319-56017-5_18

28. Holmberg S, Rignell-Hydbom A, H Lindh C, et al. High levels of vitamin $D$ associated with less ischemic heart disease - a nested case-control study among rural men in Sweden. Ann Agric Environ Med. 2017;24(2):288-293. doi: https://doi.org/10.5604/12321966.1235176

29. Welles CC, Whooley MA, Karumanchi SA, et al. Vitamin D Deficiency and Cardiovascular Events in Patients With Coronary Heart Disease: Data From the Heart and Soul Study. Am J Epidemiol. 2014;179(11):1279-1287. doi: https://doi.org/10.1093/aje/kwu059

30. Zhou J-C, Zhu Y, Gong C, et al. The GC2 haplotype of the vitamin D binding protein is a risk factor for a low plasma 25-hydroxyvitamin D concentration in a Han Chinese population. Nutr Metab (Lond). 2019;16(1):5. doi: https://doi.org/10.1186/s12986-019-0332-0

\section{ИНФОРМАЦИЯ ОБ АВТОРАХ [AUTHORS INFO]:}

* Михайлова Лариса Викторовна, к.М.Н., доцент [Larisa V. Mikhailova, MD, PhD, associate professor]; адрес: Россия, 236016, Калининград, ул. Боткина , д. 4/6 [address: 236016 Botkina street 4/6, Kaliningrad, Russia]; ORCID: https://orcid.org/0000-0001-5070-5955; eLibrary SPIN: 4991-9875; e-mail: mihalysa@mail.ru

Богачев Роберт Стефанович, д.м.н., професcop [Robert S. Bogachev, MD, PhD, professor]; ORCID: https://orcid.org/0000-0002-9651-0018; eLibrary SPIN: 3599-9607; e-mail: robcm@rambler.ru Литвинова Лариса Сергеевна, д.м.н. [Larisa S. Litvinova, MD, PhD]; ORCID: https://orcid.org/0000-0001-5070-5955; eLibrary SPIN: 4991-9875; e-mail: larisalitvinova@yandex.ru Козел Анастасия Юрьевна, студент [Anastasia Y. Kozel, student]; ORCID: https://orcid.org/0000-0002-8218-1364; eLibrary SPIN: 4991-9875; e-mail: 07.06anastasia@gmail.com Шитова Елена Сергеевна, к.M.H. [Elena S. Shytova, MD, PhD]; ORCID: https://orcid.org/0000-0001-6160-7138; eLibrary SPIN: 6896-5576; e-mail: Helenatrif@gmail.com

Анкудович Виталий Болеславович, студент [Vitaly B. Ankudovich, student];

ORCID: https://orcid.org/0000-0002-1377-3366; eLibrary SPIN: 4991-9875; e-mail: vitaliyankudovich@gmail.com Мордвинцев Владислав Валерьевич, студент [Vladislav V. Mordvintsev, student]; ORCID: https://orcid.org/0000-0002-6794-0784; eLibrary SPIN: 4991-9875; e-mail: umhic@yandex.ru

Добрынина Ульяна Андреевна, студент [Ulyana A. Dobrynina, student]; ORCID: https://orcid.org/0000-0002-4932-4255; eLibrary SPIN: 4991-9875; e-mail: Dobrynina999@gmail.com

*Авторы, ответственные за переписку. 


\section{ЦИТИРОВАТЬ:}

Богачев Р.С., Козел А.Ю., Литвинова Л.С., Михайлова Л.В., Шитова Е.С., Анкудович В.Б., Мордвинцев В.В., Добрынина У.A. Полиморфизм гена витамин D-связывающего белка у пациентов - жителей Калининградской области с острым коронарным синдромом // Ожирение и метаболизм. — 2019. - Т.16. — №3. — C.81-87. doi: https://doi.org/10.14341/omet9758

\section{TO CITE THIS ARTICLE:}

Bogachev RS, Kozel AY, Litvinova LS, Mikhailova LV, Shytova ES, Ankudovich VB, Mordvintsev VV, Dobrynina UA. Vitamin D binding protein polymorphysm in patients with acute coronary syndrome in Kaliningrad region. Obesity and metabolism. 2019;16(3):81-87. doi: https://doi.org/10.14341/omet9758 